\title{
R.E.N.A.L. nephrometry score predicts postoperative recurrence of localized renal cell carcinoma treated by radical nephrectomy
}

\author{
Akira Nagahara ${ }^{1} \cdot$ Motohide Uemura $^{1} \cdot$ Atsunari Kawashima $^{1} \cdot$ Takeshi Ujike $^{1} \cdot$ \\ Kazutoshi Fujita $^{1}$ - Yasushi Miyagawa ${ }^{1} \cdot$ Norio Nonomura $^{1}$
}

Received: 23 April 2015 / Accepted: 5 July 2015 / Published online: 29 July 2015

(C) The Author(s) 2015. This article is published with open access at Springerlink.com

\begin{abstract}
Background We investigated the association between the R.E.N.A.L. nephrometry score (RNS) and the postoperative recurrence of localized renal cell carcinoma (RCC).

Methods We retrospectively analyzed a database comprising 91 patients with non-small localized RCC (pT1b-T2b) treated by radical nephrectomy at our hospital from January 2002 to March 2010. RNS was scored based on imaging findings at diagnosis. The Cox proportional hazards model was used to predict recurrence-free survival (RFS) and to calculate hazard ratio (HR).

Results The median age at operation was 63 years (range, 30-85 years). Postoperative recurrence occurred in 19 patients (21\%). Median RNS sum was 9 (range, 5-11). High RNS sum (10-12) was significantly associated with RFS $(P=0.0012)$. Multivariate analysis revealed that high RNS sum [HR, 9.05; $95 \%$ confidence interval (CI), 2.1163.9; $P=0.0019]$ were significantly associated with RFS. Regarding each component of RNS, only the L component, which referred to tumor location relative to the polar line, was associated with RFS (HR, 15.0; $95 \%$ CI, 2.68-396; $P=0.0006)$.

Conclusions RNS was associated with RFS in cases of non-small localized RCC (pT1b-2b), thus supporting its utility as a prognostic factor.
\end{abstract}

Keywords Imaging $\cdot$ Nephrometry score $\cdot$ Radical nephrectomy $\cdot$ Recurrence $\cdot$ Renal cell carcinoma

Motohide Uemura

uemura@uro.med.osaka-u.ac.jp

1 Department of Urology, Osaka University Graduate School of Medicine, 2-2 Yamadaoka, Suita, Osaka 565-0871, Japan

\section{Introduction}

Recently, three nephrometry scoring systems, namely, the R. E. N. A. L. nephrometry score (RNS) [1], PADUA score [2], and C-index [3], all of which are based on the anatomical features of renal tumors, were developed to assess the risk of nephron-sparing surgery for a small renal mass including renal cell carcinoma (RCC). These metrics have been validated to predict perioperative complications for partial nephrectomy in many previous studies.

RNS characterizes tumors on the basis of the anatomical features of renal masses on computed tomography (CT) or magnetic resonance imaging (MRI) [1]. Of these scoring systems, only the RNS was associated with histological features of tumor aggressiveness in some reports [4-7]. Accordingly, RNS is expected to be a useful predictor of postoperative recurrence in patients with localized RCC. However, to our knowledge, there has been only one report regarding the association between RNS and postoperative recurrence [8]. For these reasons, in the present study, we decided to use RNS to analyze the association between anatomical features and postoperative recurrence after radical nephrectomy in patients with localized RCC (T1b-T2b).

\section{Patients and methods}

\section{Patients}

We retrospectively analyzed a database comprising 91 consecutive patients with localized RCC (pT1b-2b) treated by radical nephrectomy between January 2002 and March 2013 at the Osaka University Hospital. Staging of RCC was performed according to the 7th edition of TNM staging proposed by the International Union for Cancer Control 
and the American Joint Committee on Cancer [9]. RNS (www.nephrometry.com) consists of R, E, N, A, and L components based on the following anatomical features of RCC: size, endophytic degree, proximity to the collecting system or renal sinus, position (anterior or posterior), and location relative to the polar line, respectively. If the tumor is in contact with the main renal artery or vein, the suffix $\mathrm{h}$ is assigned. RNS was calculated on the basis of findings of preoperative CT or MRI. Recurrence-free survival (RFS) time was calculated from the date of surgery until the date of recurrence or the date of the patient's last follow-up visit. The study was approved by the institutional review board of Osaka University hospital (approval number: 11397-2).

\section{Statistical analysis}

RFS rate was calculated using the Kaplan-Meier method, and comparisons were made using the log-rank test. The median value of each RNS parameter was used for the cutoff value. For preoperative serum C-reactive protein (CRP) level, $0.2 \mathrm{mg} / \mathrm{dl}$ was used for cutoff value. Univariate and multivariate analyses were performed using the COX proportional hazards model to predict RFS and calculate hazard ratio. Variables entered into the model for RFS analysis included patient age, gender, preoperative serum CRP level, histological grade, histological type, microscopic vascular involvement, RNS sum, and each component of RNS. The variables that had a $P$ value $<0.1$ in the univariate analysis were entered into the multivariate analysis. Two kinds of multivariate analysis, one including the RNS sum and the other including each score-constituent parameter of RNS, were performed. All statistical analyses were performed using JMP Pro version 11 (SAS Institute, Tokyo, Japan), with $P<0.05$ considered as statistically significant.

\section{Results}

The clinical and pathological characteristics of the 91 patients with localized RCC and their treatment outcomes are shown in Table 1 . The median age at operation was 63 years (range, 30-85 years). The histological type of RCC of 80 patients was clear cell carcinoma and the remaining 11 were non-clear cell carcinoma. Postoperative recurrence occurred in 19 patients $(21 \%)$, and the median time to cancer recurrence after radical nephrectomy was 27 months (range, 1-79 months). The recurrence sites were as follows: lung (10 patients, $53 \%$ ), contralateral kidney ( 2 patients, $11 \%$ ), bone ( 1 patient, $5 \%$ ), contralateral ureter ( 1 patient, $5 \%$ ), gallbladder ( 1 patient, $5 \%$ ), pancreas ( 1 patient, $5 \%$ ), small intestine (1 patient, $5 \%$ ), hilar lymph node (1 patient, $5 \%$ ), and mediastinal lymph node
Table 1 Clinicopathological characteristics of the 91 patients

\begin{tabular}{|c|c|c|}
\hline Characteristics & Total & $(n=91)$ \\
\hline Age, years (median) & $30-85(63)$ & \\
\hline \multicolumn{3}{|l|}{ Gender } \\
\hline Male & 59 & $(65 \%)$ \\
\hline Female & 32 & $(35 \%)$ \\
\hline \multicolumn{3}{|l|}{$\mathrm{R} / \mathrm{L}$} \\
\hline Right & 44 & $(48 \%)$ \\
\hline Left & 47 & $(52 \%)$ \\
\hline \multicolumn{3}{|l|}{ Preoperative serum CRP level } \\
\hline$\leq 0.2 \mathrm{mg} / \mathrm{dl}$ & 72 & $(79 \%)$ \\
\hline$>0.2 \mathrm{mg} / \mathrm{dl}$ & 18 & $(20 \%)$ \\
\hline Unknown & 1 & $(1 \%)$ \\
\hline \multicolumn{3}{|l|}{ pT stage } \\
\hline $1 \mathrm{~b}$ & 65 & $(71 \%)$ \\
\hline $2 \mathrm{a}$ & 18 & $(20 \%)$ \\
\hline $2 \mathrm{~b}$ & 8 & $(9 \%)$ \\
\hline \multicolumn{3}{|l|}{ Histological type } \\
\hline Clear cell carcinoma & 80 & $(88 \%)$ \\
\hline Non-clear cell carcinoma & 11 & $(12 \%)$ \\
\hline \multicolumn{3}{|l|}{ Histological grade } \\
\hline 1 & 5 & $(5 \%)$ \\
\hline 2 & 73 & $(80 \%)$ \\
\hline 3 & 13 & $(14 \%)$ \\
\hline \multicolumn{3}{|c|}{ Microscopic vascular involvement } \\
\hline Absent & 82 & $(90 \%)$ \\
\hline Present & 5 & $(5 \%)$ \\
\hline Unknown & 4 & $(4 \%)$ \\
\hline \multicolumn{3}{|l|}{ Recurrence } \\
\hline No & 72 & $(79 \%)$ \\
\hline Yes & 19 & $(21 \%)$ \\
\hline Follow up, months (median) & $1-156(65)$ & \\
\hline
\end{tabular}

(1 patient, $5 \%)$. In addition, 3 patients $(16 \%)$ with cancer recurrence died of RCC progression during the observation periods. The median follow-up period was 65 months (range, 1-156 months). Anatomic characteristics based on RNS are shown in Table 2.

Figure 1 shows the Kaplan-Meier curve of RFS rate stratified according to RNS sum (4-9 vs. 10-12). RFS rate in patients with a low RNS sum was much better than that in patients with a high RNS sum $(P=0.0012)$. The 5- and 10 -year RFS rates in patients with high RNS sum $(70 \%$ and $55 \%$, respectively) were significantly worse than those in patients with low RNS sum (95\% and $95 \%$, respectively). Figure 2 shows the Kaplan-Meier curve of the RFS rate stratified according to each component of RNS. RFS rate was significantly different when stratified by the $\mathrm{R}$ component $(P=0.0009)$, the $\mathrm{L}$ component $(P=0.0001)$, and the presence of hilar tumor $(P=0.0396)$. 
Table 2 Anatomic characteristics as based on R.E.N.A.L. nephrometry score

\begin{tabular}{|c|c|c|}
\hline Characteristics & Total & $(n=91)$ \\
\hline \multicolumn{3}{|c|}{ R.E.N.A.L. nephrometry score sum } \\
\hline $4-6$ & 2 & $(2 \%)$ \\
\hline $7-9$ & 44 & $(48 \%)$ \\
\hline $10-12$ & 45 & $(49 \%)$ \\
\hline \multicolumn{3}{|l|}{$\mathrm{R}$ component } \\
\hline 1 & 0 & $(0 \%)$ \\
\hline 2 & 60 & $(66 \%)$ \\
\hline 3 & 31 & $(34 \%)$ \\
\hline \multicolumn{3}{|l|}{ E component } \\
\hline 1 & 33 & $(36 \%)$ \\
\hline 2 & 53 & $(58 \%)$ \\
\hline 3 & 5 & $(5 \%)$ \\
\hline \multicolumn{3}{|l|}{$\mathrm{N}$ component } \\
\hline 1 & 2 & $(2 \%)$ \\
\hline 2 & 2 & $(2 \%)$ \\
\hline 3 & 87 & $(96 \%)$ \\
\hline \multicolumn{3}{|l|}{ A component } \\
\hline $\mathrm{a}$ & 22 & $(24 \%)$ \\
\hline $\mathrm{p}$ & 17 & $(19 \%)$ \\
\hline $\mathrm{x}$ & 52 & $(57 \%)$ \\
\hline \multicolumn{3}{|l|}{ L component } \\
\hline 1 & 15 & $(16 \%)$ \\
\hline 2 & 29 & $(32 \%)$ \\
\hline 3 & 47 & $(52 \%)$ \\
\hline \multicolumn{3}{|l|}{ Presence of $h$} \\
\hline $\mathrm{h}(-)$ & 49 & $(54 \%)$ \\
\hline $\mathrm{h}(+)$ & 42 & $(46 \%)$ \\
\hline
\end{tabular}

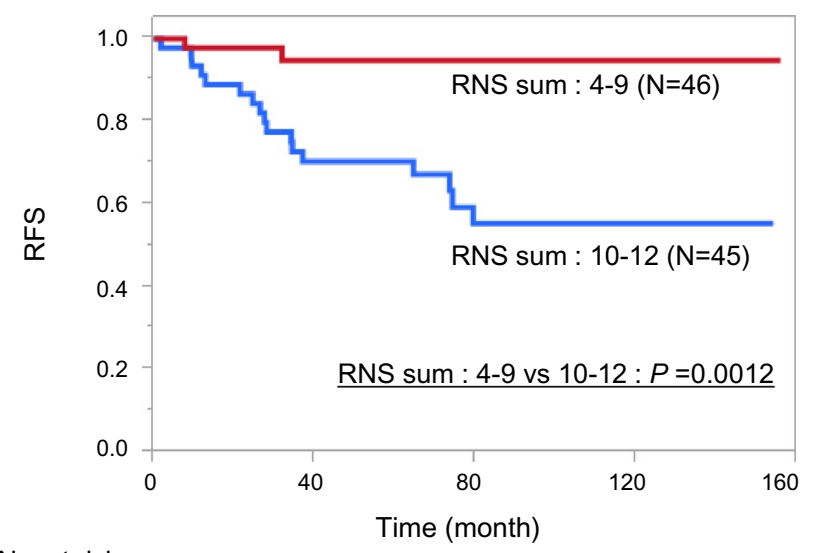

No. at risk

$\begin{array}{llllll}\text { 4-9: } & 40 & 25 & 12 & 2 & 0 \\ \text { 10-12: } & 45 & 28 & 14 & 4 & 0\end{array}$

Fig. 1 Probability estimates of recurrence-free survival (RFS) rate in 91 patients in two groups based on R.E.N.A.L. nephrometry score (RNS) sum (4-9 vs. 10-12)
The results of univariate analysis are shown in Table 3. Of several factors examined, RNS sum, R component, $\mathrm{L}$ component, and the presence of $\mathrm{h}$ were significant factors in predicting postoperative cancer recurrence. Age at operation, preoperative serum CRP level and A component ( $\mathrm{x}$ vs. p) were weakly associated with RFS rate on univariate analysis. The multivariate analysis including age at operation, preoperative serum CRP level, RNS sum, A component, and presence of h showed that only the RNS sum (HR, 9.05; $95 \%$ CI, 2.11-63.9; $P=0.0019$ ) was an independent predictive factor for postoperative cancer recurrence (Table 3). A second multivariate analysis replacing RNS sum with the R and L components, which were significant score-constituent factors of RNS on univariate analysis, showed that the L component (HR, 15.0; $95 \%$ CI, 2.68-285; $P=0.0006)$ was an independent predictor of postoperative cancer recurrence (Table 3 ). Of the components of RNS, only the L component was significantly associated with RFS rate.

\section{Discussion}

RNS was developed by Kutikov and Uzzo to standardize the assessment of anatomical features of renal tumors [1]. RNS consists of (R)adius (tumor size at maximal diameter), (E)xophytic/endophytic properties of the tumor, $(\mathrm{N})$ earness of the deepest portion of tumor to the collecting system or sinus, (A)nterior (a)/posterior (p) descriptor, and the (L)ocation relative to the polar line. The suffix h (hilar) is assigned to tumors that are close to the main renal artery or vein. RNS is scored based on CT or MRI findings at diagnosis.

Because RNS was developed to quantitatively evaluate the complexity of renal tumors, many studies confirmed the usefulness of RNS for predicting perioperative outcomes in patients treated by partial nephrectomy [10-15]. Recently, some studies revealed a relationship between RNS and histological features of tumor aggressiveness [4-7]. Kutikov et al. compared the individual components of RNS with histology and grade of 525 resected tumors and constructed a novel nomogram for predicting high-grade histology. In their analyses, high $\mathrm{R}$ and $\mathrm{L}$ scores were strongly associated with high-grade histology [4]. Wang et al. validated this nomogram in 391 Chinese RCC patients [5]. Mullins et al. revealed a high RNS sum was associated with highgrade pathology in a study of 886 patients treated by robotassisted partial nephrectomy [7].

The present study evaluated the relationship between RNS and postoperative cancer recurrence of non-small localized RCC (pT1b-pT2b). A high RNS sum was significantly associated with low RFS rate $(P=0.0012)$, and the RNS sum was a significant, independent factor 


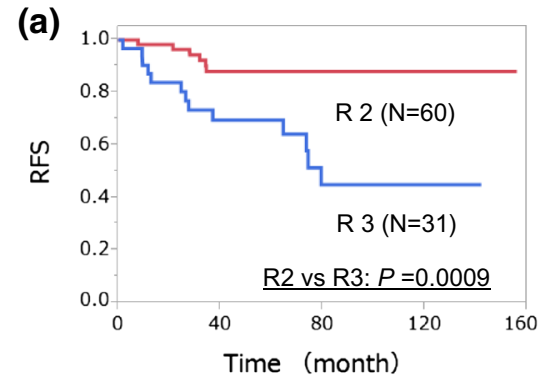

No. at risk

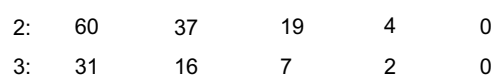

(d)

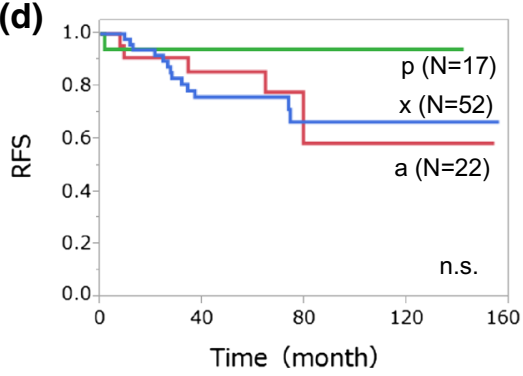

No. at risk

$\begin{array}{llllll}\text { p: } & 17 & 11 & 9 & 1 & 0 \\ \text { x: } & 52 & 27 & 14 & 4 & 0 \\ \text { a: } & 22 & 15 & 3 & 1 & 0\end{array}$

(b)

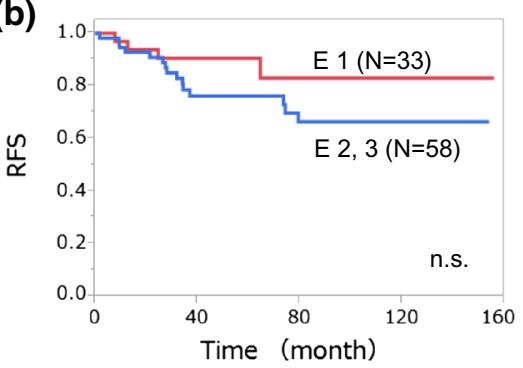

No. at risk

1: $\begin{array}{llllll}33 & 20 & 6 & 2 & 0\end{array}$

(c)

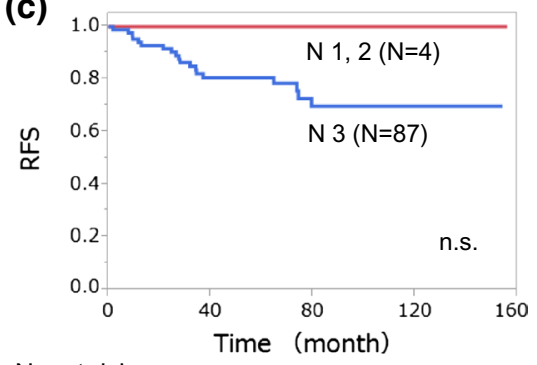

No. at risk

$\begin{array}{llllll}\text { 1, 2: } & 4 & 3 & 2 & 2 & 0 \\ \text { 3: } & 87 & 50 & 24 & 4 & 0\end{array}$

(f)

(e)

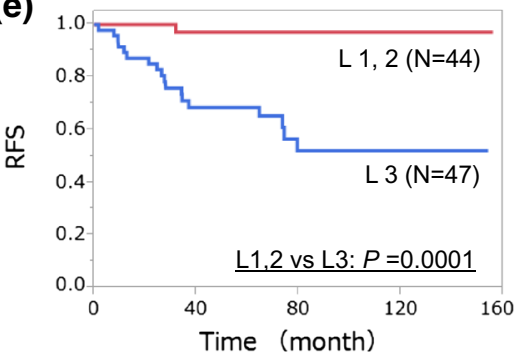

No. at risk

1, 2: $44 \quad 27 \quad 14 \quad 2 \quad 0$

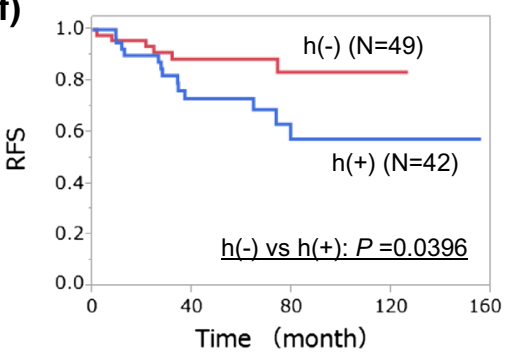

No. at risk

$\begin{array}{llllll}\mathrm{h}(-): & 49 & 30 & 16 & 1 & 0\end{array}$

$h(+): \quad 42 \quad 23 \quad 10 \quad 5 \quad 0$
Fig. 2 Probability estimates of recurrence-free survival rate $(R F S)$ in 91 patients stratified based on $\mathrm{R}$ component (score: 2 vs. 3) (a), E component (score: 1 vs. 2, 3) (b), N component (score: 1, 2 vs. 3) (c),
A component (a vs. p vs. x) (d), L component (score: 1, 2 vs. 3) (e), and presence of $\mathrm{h}$ (absent vs. present) (f)

Table 3 Cox proportional hazards analysis of R.E.N.A.L. nephrometry score and clinicopathological factors to predict recurrence-free survival

\begin{tabular}{|c|c|c|c|c|c|c|}
\hline \multirow[t]{2}{*}{ Characteristics } & \multicolumn{2}{|l|}{ Univariate } & \multicolumn{2}{|l|}{ Multivariate 1} & \multicolumn{2}{|l|}{ Multivariate 2} \\
\hline & HR $(95 \%$ CI $)$ & $P$ value & HR (95 \% CI) & $P$ value & HR (95\% CI) & $P$ value \\
\hline Age (years) (continuous) & $1.04(1.00-1.08)$ & 0.0753 & $1.04(1.00-1.08)$ & 0.0739 & $1.03(0.99-1.08)$ & 0.0619 \\
\hline Gender (male vs. female) & $1.34(0.53-3.84)$ & 0.5404 & & & & \\
\hline Preoperative serum CRP level ( $>0.2$ vs. $\leq 0.2 \mathrm{mg} / \mathrm{dl}$ ) & $2.39(0.83-6.13)$ & 0.0996 & $2.39(0.80-6.51)$ & 0.1114 & $2.82(0.91-8.26)$ & 0.0710 \\
\hline Histological grade (G1, G2 vs. G3) & $1.24(0.29-3.75)$ & 0.7378 & & & & \\
\hline Histological type (clear cell vs. non-clear cell) & $1.13(0.32-7.11)$ & 0.8707 & & & & \\
\hline Microscopic vascular involvement (+ vs. - ) & $2.78(0.44-9.83)$ & 0.2306 & & & & \\
\hline R.E.N.A.L. nephrometry score sum (10-12 vs. 4-9) & $7.77(2.22-49.0)$ & 0.0005 & $9.05(2.11-63.9)$ & 0.0019 & & \\
\hline \multicolumn{7}{|l|}{ R.E.N.A.L. nephrometry score component } \\
\hline R (3 vs. 2) & $4.49(1.77-12.8)$ & 0.0014 & & & $2.53(0.96-7.62)$ & 0.0619 \\
\hline $\mathrm{E}(2,3$ vs. 1$)$ & $1.99(0.72-7.00)$ & 0.1982 & & & & \\
\hline $\mathrm{N}(3$ vs. 1,2$)$ & $4.69(0.06-401)$ & 0.496 & & & & \\
\hline $\mathrm{L}(3$ vs. 1,2$)$ & $17.49(3.61-315)$ & $<0.0001$ & & & $15.0(2.68-285)$ & 0.0006 \\
\hline A (a vs. p) & $4.48(0.72-86.4)$ & 0.1165 & $3.19(0.48-62.7)$ & 0.2520 & $2.57(0.39-50.3)$ & 0.3536 \\
\hline A (x vs. p) & $4.88(0.97-88.8)$ & 0.0562 & $2.69(0.51-49.6)$ & 0.2859 & $2.15(0.39-40.2)$ & 0.4314 \\
\hline Presence of h (+ vs. -$)$ & $2.66(1.05-7.57)$ & 0.039 & $0.85(0.30-2.74)$ & 0.7754 & $0.75(0.26-2.32)$ & 0.6043 \\
\hline
\end{tabular}


for predicting postoperative cancer recurrence by multivariate analysis. Of the RNS components, only the L component was strongly associated with RFS rate. Kopp et al. revealed high RNS sum (10-12) and transfusion status were associated with shorter progression-free survival in a study of 202 patients with localized cT2 renal masses treated by radical nephrectomy or partial nephrectomy [8]. They also revealed high RNS sum, high nuclear grade, and transfusion status were associated with shorter overall survival. To date, there has been only one report suggesting that RNS was a predictive factor for postoperative cancer recurrence. The present study is the first to our knowledge that demonstrates a relationship between RNS, especially the L component, and postoperative recurrence.

Many predictive factors of recurrence after surgery in patients with RCC have been reported in the literature, and of anatomical tumor characteristics, tumor size has been identified as predictive of recurrence in many studies [16-18]. In the present study, the R component, namely, tumor size, was significantly associated with RFS rate on univariate analysis. However, upon multivariate analysis using all components of RNS, only the L component was significantly associated with RFS, whereas all other components were not significant, including R. To our knowledge, no other study has reported a similar result, making our current study unique in its findings. A study by Matsumoto et al. revealed a correlation between RNS and annual growth rates of renal masses scheduled for active surveillance [19], in which only the L component was significantly correlated with annual growth rate by multivariate analysis. These findings suggest that biological aggressiveness may be influenced by tumor location, although the underlying mechanism for this concept has not yet been fully clarified.

Among several histological characteristics, nuclear grade, presence of microvascular invasion, and tumor necrosis have been reported as predictive factors for postoperative cancer recurrence [16, 17, 20]. Of clinical and biochemical features, age at diagnosis, performance status, and the serum level of CRP have been reported as predictors of postoperative cancer recurrence [18, 20-23]. Several interesting studies have reported on molecular predictors of postoperative recurrence. Shvarts et al. reported that p53 expression in the tumor was related to nuclear grade and significantly associated with RFS rate in patients with localized RCC treated by radical nephrectomy [22]. Recently, Fujita et al. revealed that the level of vascular endothelial growth factor in preoperative serum was an independent predictor of postoperative recurrence in localized clear cell RCC [24]. Hongo et al. also revealed that cyclin-dependent kinase-related parameters were strongly associated with recurrence after surgery [25].
The present study had some limitations. First, the patient cohort of this study was small, and their $\mathrm{T}$ stage ranged from $\mathrm{T} 1 \mathrm{~b}$ to $\mathrm{T} 2 \mathrm{~b}$. Considering the increasing incidence of T1astage tumors among RCC cases, the results of the present study may not perfectly represent localized RCC. A large study including T1a disease may produce more reliable results, but because cancer recurrence in patients with $\mathrm{T} 1 \mathrm{a}$ $\mathrm{RCC}$ is rare, such a study may require a large number of patients to reach statistical significance. Second, as already mentioned, although many clinicopathological and novel molecular predictors of postoperative recurrence have been reported, our study utilized only a small number of clinicopathological parameters. Further studies would be needed to fully assess the relevance of other known predictive factors in evaluating the prognostic value of RNS on RFS rate.

In conclusion, the present study showed that RNS sum was independent predictors of postoperative recurrence in patients with non-small localized RCC (pT1b-T2b) treated by radical nephrectomy. Of the RNS components, only the L component was significantly associated with RFS. Accurate prediction of recurrence after surgical resection would be highly valuable in designing adjuvant trials, for example, in immunotherapy or molecular target therapy trials, and also for effectively scheduling follow-up visits and imaging studies.

\section{Compliance with ethical standards}

Conflict of interest The authors declare that they have no conflict of interest.

Open Access This article is distributed under the terms of the Creative Commons Attribution 4.0 International License (http://creativecommons.org/licenses/by/4.0/), which permits unrestricted use, distribution, and reproduction in any medium, provided you give appropriate credit to the original author(s) and the source, provide a link to the Creative Commons license, and indicate if changes were made.

\section{References}

1. Kutikov A, Uzzo RG (2009) The R.E.N.A.L. nephrometry score: a comprehensive standardized system for quantitating renal tumor size, location and depth. J Urol 182:844-853

2. Ficarra V, Novara G, Secco S et al (2009) Preoperative aspects and dimensions used for an anatomical (PADUA) classification of renal tumours in patients who are candidates for nephronsparing surgery. Eur Urol 56:786-793

3. Simmons MN, Ching CB, Samplaski MK et al (2010) Kidney tumor location measurement using the $\mathrm{C}$ index method. $\mathrm{J}$ Urol 183:1708-1713

4. Kutikov A, Smaldone MC, Egleston BL et al (2011) Anatomic features of enhancing renal masses predict malignant and highgrade pathology: a preoperative nomogram using the RENAL Nephrometry score. Eur Urol 60:241-248 
5. Wang H-K, Zhu Y, Yao X-D et al (2012) External validation of a nomogram using RENAL nephrometry score to predict high grade renal cell carcinoma. J Urol 187:1555-1560

6. Satasivam P, Sengupta S, Rajarubendra N et al (2012) Renal lesions with low R.E.N.A.L nephrometry score are associated with more indolent renal cell carcinomas (RCCs) or benign histology: findings in an Australian cohort. BJU Int 109(Suppl):44-47

7. Mullins JK, Kaouk JH, Bhayani S et al (2012) Tumor complexity predicts malignant disease for small renal masses. J Urol 188:2072-2076

8. Kopp RP, Mehrazin R, Palazzi KL et al (2014) Survival outcomes after radical and partial nephrectomy for clinical T2 renal tumours categorised by R.E.N.A.L. nephrometry score. BJU Int 114:708-718

9. Edge SB, Compton CC (2010) The American Joint Committee on Cancer: the 7th edition of the AJCC cancer staging manual and the future of TNM. Ann Surg Oncol 17:1471-1474

10. Miyake H, Furukawa J, Hinata N et al (2014) Significant impact of R.E.N.A.L. nephrometry score on changes in postoperative renal function early after robot-assisted partial nephrectomy. Int J Clin Oncol 20:586-592

11. Long J-A, Arnoux V, Fiard G et al (2013) External validation of the RENAL nephrometry score in renal tumours treated by partial nephrectomy. BJU Int 111:233-239

12. Liu Z-W, Olweny EO, Yin G et al (2013) Prediction of perioperative outcomes following minimally invasive partial nephrectomy: role of the R.E.N.A.L nephrometry score. World J Urol 31:1183-1189

13. Hayn MH, Schwaab T, Underwood W, Kim HL (2011) RENAL nephrometry score predicts surgical outcomes of laparoscopic partial nephrectomy. BJU Int 108:876-881

14. Rosevear HM, Gellhaus PT, Lightfoot AJ et al (2012) Utility of the RENAL nephrometry scoring system in the real world: predicting surgeon operative preference and complication risk. BJU Int 109:700-705

15. Altunrende F, Laydner H, Hernandez AV et al (2013) Correlation of the RENAL nephrometry score with warm ischemia time after robotic partial nephrectomy. World J Urol 31:1165-1169
16. Leibovich BC, Blute ML, Cheville JC et al (2003) Prediction of progression after radical nephrectomy for patients with clear cell renal cell carcinoma: a stratification tool for prospective clinical trials. Cancer (Phila) 97:1663-1671

17. Sorbellini M, Kattan MW, Snyder ME et al (2005) A postoperative prognostic nomogram predicting recurrence for patients with conventional clear cell renal cell carcinoma. J Urol 173:48-51

18. Muramaki M, Miyake H, Sakai I et al (2011) Age at diagnosis as a powerful predictor for disease recurrence after radical nephrectomy in Japanese patients with pT1 renal cell carcinoma. Int J Urol 18:121-125

19. Matsumoto $\mathrm{R}$, Abe $\mathrm{T}$, Shinohara $\mathrm{N}$ et al (2014) RENAL nephrometry score is a predictive factor for the annual growth rate of renal mass. Int J Urol 21:549-552

20. Ramsey S, Lamb GW, Aitchison M, McMillan DC (2008) Prospective study of the relationship between the systemic inflammatory response, prognostic scoring systems and relapse-free and cancer-specific survival in patients undergoing potentially curative resection for renal cancer. BJU Int 101:959-963

21. Jeong IG, Yoo CH, Song $\mathrm{K}$ et al (2009) Age at diagnosis is an independent predictor of small renal cell carcinoma recurrencefree survival. J Urol 182:445-450

22. Shvarts O, Seligson D, Lam J et al (2005) P53 is an independent predictor of tumor recurrence and progression after nephrectomy in patients with localized renal cell carcinoma. J Urol 173:725-728

23. Ito K, Yoshii H, Sato A et al (2011) Impact of postoperative $\mathrm{C}$-reactive protein level on recurrence and prognosis in patients with NOM0 clear cell renal cell carcinoma. J Urol 186:430-435

24. Fujita N, Okegawa T, Terado Y et al (2014) Serum level and immunohistochemical expression of vascular endothelial growth factor for the prediction of postoperative recurrence in renal cell carcinoma. BMC Res Notes 7:369

25. Hongo F, Takaha N, Oishi $\mathrm{M}$ et al (2014) CDK1 and CDK2 activity is a strong predictor of renal cell carcinoma recurrence. Urol Oncol 32:1240-1246 